\title{
ON BANACH-MAZUR COMPACTA
}

\author{
SERGEI M. AGEEV and DUŠAN REPOVŠ
}

(Received 30 November 1998; revised 27 March 2000)

Communicated by J. A. Hillman

\begin{abstract}
We study Banach-Mazur compacta $Q(n)$, that is, the sets of all isometry classes of $\boldsymbol{n}$-dimensional Banach spaces topologized by the Banach-Mazur metric. Our main result is that $Q(2)$ is homeomorphic to the compactification of a Hilbert cube manifold by a point, for we prove that $Q_{c}(2)=Q(2) \backslash\{$ Eucl. $\}$ is a Hilbert cube manifold. As a corollary it follows that $Q(2)$ is not homogeneous.

2000 Mathematics subject classification: primary 57S10; secondary 54C55, 54F45.

Keywords and phrases: noncompact Lie group, approximate $G-A N E$ space, convex $G$-space, proper $G$-space, Banach-Mazur compactum.
\end{abstract}

\section{Introduction}

This paper studies topological properties of Banach-Mazur compacta $Q(n)$, that is, the sets of all isometry classes of $n$-dimensional Banach spaces topologized by the Banach-Mazur metric. Recently, substantial progress was made concerning these spaces. It was proved in [14] that $Q(2)$ is an absolute extensor (defined below). Later this result was generalized to all $n \geq 2$ (see [5]). The long-standing problem about topological equivalence of $Q(n)$ and the Hilbert cube $I^{\infty}$ was finally solved negatively for $n=2$ in [4].

THEOREM 1.1. $Q(2)$ and $I^{\infty}$ are not homeomorphic.

For any space $X$ to be homeomorphic to the Hilbert cube $I^{\infty}$, the following necessary conditions must be satisfied for every point $x \in X$ :

(a) $X \backslash\{x\}$ must be homotopically trivial; and

(b) $X \backslash\{x\}$ must be a Hilbert cube manifold.

(c) 2000 Australian Mathematical Society $0263-6115 / 2000 \$ A 2.00+0.00$ 
The key idea of the proof of Theorem 1.1 was to show that $Q(2)$ fails to possess the property (a) at the Euclidean point \{Eucl.\}, which corresponds to the isometry class of the Euclidean space. On the other hand, the main result of this paper, Theorem 1.2 stated below, implies that the complement $Q(2) \backslash\{x\}$ of every other point $x \in Q(2)$ turns out to be homotopically trivial. Furthermore, Theorem 1.2 demonstrates that as far as the property (b) is concerned, everything turns out to be exactly the opposite: $Q(2) \backslash\{$ Eucl. $\}$ is a Hilbert cube manifold, while the complement $Q(2) \backslash\{x\}$ of every other point $x \in Q(2)$ is not.

THEOREM 1.2. $Q_{\delta}(2)=Q(2) \backslash\{$ Eucl. $\}$ is a Hilbert cube manifold.

As a corollary we prove that $Q(2)$ is not homogeneous (recall that a space $X$ is said to be homogeneous if for every pair of points $x_{1}, x_{2} \in X$ there exists a homeomorphism $h: X \rightarrow X$ such that $\left.h\left(x_{1}\right)=x_{2}\right)$.

COROLLARY 1.3. $Q(2)$ is not a homogeneous space.

ProOF OF COROLlaRY 1.3. By [4], [Eucl.] is not a $Z$-set in $Q(2)$. On the other hand, it follows by our Theorem 1.2 above that for every point $x \in Q(2) \backslash\{$ Eucl. $\},\{x\}$ is a $Z$-set in $Q(2) \backslash\{$ Eucl. $\}$, hence also a $Z$-set in $Q(2)$. Therefore $(Q(2),\{$ Eucl. $\}) \neq$ $(Q(2),\{x\})$.

\section{Preliminaries}

We identify the set $\operatorname{BAN}(n)$ of all $n$-dimensional Banach spaces with the set of all norms in $\mathbb{R}^{n}$. The Banach-Mazur distance $\rho(X, Y)$ between spaces $X=\left\{\mathbb{R}^{n},\|\cdot\|_{X}\right\}$ and $Y=\left\{\mathbb{R}^{n},\|\cdot\|_{Y}\right\} \in \operatorname{BAN}(n)$ is defined as follows:

$$
\rho(X, Y)=\inf \left\{\|T\| \cdot\left\|T^{-1}\right\| \mid T: X \rightarrow Y \text { is an isomorphism }\right\},
$$

where $\|T\|,\left\|T^{-1}\right\|$ are norms of the operators $T$ and $T^{-1}$, respectively. It is well-known that for every $X, Y, Z \in \mathrm{BAN}(n)$, the following properties hold:

(1) $\rho(X, Z) \leq \rho(X, Y) \rho(Y, Z)$;

(2) $\rho(X, Y)=\rho(Y, X) \geq 1$; and

(3) $\rho(X, Y)=1$ if and only if $X$ and $Y$ are isometric, $X \cong Y$, that is, there exists an isomorphism $T: X \rightarrow Y$ which preserves the norm $\|x\|_{X}=\|T(x)\|_{Y}$ for every $x \in X$.

It follows that the function $\ln \rho(X, Y)$ is a pseudometric on the space $\operatorname{BAN}(n)$, which in the decomposition space $Q(n)=\operatorname{BAN}(n) / \cong$ becomes the metric $d([X],[Y])$ $=\ln \rho(X, Y)$, where

$$
X \cong Y \Longleftrightarrow \rho(X, Y)=1 \Longleftrightarrow \ln \rho(X, Y)=0 .
$$


The resulting metric space $(Q(n), d)$ of all isometry classes of $n$-dimensional Banach spaces is called the Banach-Mazur compactum.

This compactum allows for a different, more suitable presentation as a decomposition of the space $C(n)$ of all compact convex symmetric (rel 0$)$ bodies in $\mathbb{R}^{n}$. If one measures the distance between subsets of $\mathbb{R}^{n}$ by the Hausdorff metric $\rho_{H}(A, B)$ and defines the linear combination $\sum_{i=0}^{n} \lambda_{i} A_{i}$ by means of the Minkowski operation, then $\left(C(n), \rho_{H}\right)$ becomes a locally compact convex space.

Moreover, $C(n)$ can be equipped with an action of the general linear group $\mathrm{GL}(n) \times$ $C(n) \rightarrow C(n)$, given by $T \cdot V=T(V)$, where $T: \mathbb{R}^{n} \rightarrow \mathbb{R}^{n} \in \mathrm{GL}(n)$ and $V \in C(n)$, which agrees with the convex structure on $C(n)$. We show that the orbit space $C(n) / \mathrm{GL}(n)$ is naturally homeomorphic to the Banach-Mazur compactum.

Indeed, for an arbitrary body $V \in C(n)$, the Minkowski functional $p_{V}(x)=$ $\inf \left\{t^{-1} \mid t x \in V\right\}$ defines a norm on $\mathbb{R}^{n}$ and consequently, induces a continuous bijection $M: C(n) \rightarrow \operatorname{BAN}(n)$ defined by $M(V)=\left(\mathbb{R}^{n}, p_{V}\right)$. Since it is well-known that Banach spaces $M(V)$ and $M(W)$ are isomorphic if and only if $V=T \cdot W$ for some $T \in \mathrm{GL}(n)$, it follows that $M$ induces a continuous bijection of the quotient spaces

$$
\tilde{M}: C(n) / \mathrm{GL}(n) \rightarrow Q(n)=\mathrm{BAN}(n) / \cong,
$$

which is a homeomorphism.

Hereafter, we shall consider only locally compact Lie groups (for example GL $(n)$ ), metric spaces and continuous maps, unless otherwise specified. An action of $G$ on a space $X$ is a homeomorphism $T: G \rightarrow$ Aut $X$ of the group $G$ into the group Aut $X$ of all autohomeomorphisms of $X$ such that the map $G \times X \rightarrow X$, given by $(g, x) \mapsto T(g)(x)=g x$, is continuous. A space $X$ with a fixed action of $G$ is called a $G$-space.

For any point $x \in X$, the isotropy subgroup of $x$, or the stabilizer of $x$, is defined as $G_{x}=\{g \in G \mid g x=x\}$, and the orbit of $x$ as $G(x)=\{g x \mid g \in G\}$. The space of all orbits is denoted by $X / G$ and the natural map $\pi: X \rightarrow X / G$, given by $\pi(x)=G(x)$, is called the orbit projection. The orbit space $X / G$ is equipped with the quotient topology, induced by $\pi$.

Actions of noncompact groups $G$ do not agree very well with the orbit structure of $X$ : the orbit of a point $x$ can be dense in $X$, the orbit space $X / G$ can be non-Hausdorff, two orbits with the same stabilizer can be nonhomeomorphic, etc. Palais [22] singled out a class of $G$-spaces with the action of a locally compact group which do not have such deficiencies-he called such spaces proper.

DEFINITION 2.1. (a) Given subsets $A, B \subset X$ consider the following subset of the group $G$ :

$$
((A, B))=\{g \in G \mid g A \cap B \neq \emptyset\} .
$$


Then $A$ is said to be thin with respect to $B$, if $((A, B))$ is precompact, that is, it lies in a compact subset of $G$. Since $((A, B))=((B, A))^{-1}$, it follows that $B$ is also thin with respect to $A$.

(b) $A \subset X$ is said to be small if for every point $x \in X$, there exists a neighbourhood $O(x) \subset X$ of $x$, which is thin with respect to $A$.

(c) A $G$-space $X$ is said to be proper if it possesses a basis, consisting of small neighbourhoods.

In general, the orbit projection $\pi: X \rightarrow X / G$ of a proper $G$-space $X$ fails to be a closed map. This forces us to seek those closed subsets $F \subset X$ of $X$ for which the restriction $\left.\pi\right|_{F}: F \rightarrow X / G$ is closed.

DEFINITION 2.2. A closed subset $F \subset Z$ of a $G$-space $Z$ is said to be fundamental if $F$ is small in $Z$ and intersects every orbit, that is, $F \cap G(z) \neq \emptyset$ for every $z \in Z$.

PROPOSITION 2.3. Suppose that a G-space $Z$ is proper and that the orbit space $Z / G$ is metrizable. Then

(d) there exists a fundamental subset $F \subset Z$; and

(e) for every fundamental subset $F \subset Z$, the restriction $\left.\pi\right|_{F}: F \rightarrow Z / G$ is a proper map.

DEFINITION 2.4. An exact slice at the point $x \in X$ is a $G$-map $\varphi: U \rightarrow G(x)$ of some $G$-neighbourhood $U \subset X$ (that is, $G \cdot U=U$ ) of the orbit $G(x)$, such that $\varphi(x)=x$. The preimage $\varphi^{-1}(x)$ of the point $x$ is also called a slice or a $G_{x}$-kernel.

The principal results concerning slices belong to Abels [1] and Palais [22].

THEOREM 2.5 (Palais). A proper completely regular G-space X has a slice at every point $x$.

THEOREM 2.6 (Abels). Let $X$ be a proper $G$-space with a paracompact orbit space and $K$ a maximal compact subgroup of $G$. Then there exists a $G$-map $f: X \rightarrow G / K$ (a so-called global $K$-slice). Conversely, if there exists a global $K$-slice, then $X$ is a proper G-space.

In the sequel, we shall work in the class $\mathscr{G}$ of all metric proper $G$-spaces, whose orbit space is also metric. The following properties of the class $\mathscr{G}$ are well known (see [22]).

PROPOSITION 2.7. Let $X \in \mathscr{G}$ and let $Y$ be a separable metric $G$-space. Then the following properties hold: 
(f) The orbit $G(x)$ is closed in $X$, the stabilizer $G_{x}$ is compact and the natural map $G / G_{x} \rightarrow G(x)$, given by $g \cdot G_{x} \mapsto g x$, is a homeomorphism.

(g) $X$ can be equipped with an invariant metric, that is, $d\left(g x, g x^{\prime}\right)=d\left(x, x^{\prime}\right)$, for every $g \in G$ and $X \times Y \in \mathscr{G}$.

(h) $G / L \in \mathscr{G}$, for every compact subgroup $L<G$.

Next, we introduce several notions connected with the property of absolute extendability of maps. A space $X$ is called an absolute neighbourhood extensor, $X \in A N E$, if every map $\varphi: A \rightarrow X$, defined on a closed subset $A \subset Z$ of a metric space $Z$, and called a partial map, can be extended over some neighbourhood $U \subset Z$ of $A$, $\hat{\varphi}: U \rightarrow X,\left.\hat{\varphi}\right|_{A}=\varphi$. If we can always take $U=Z$, then $X$ is called an absolute extensor, $X \in A E$. We note that in the case when $X$ is a metric space, the concepts of the absolute (neighbourhood) retract and the absolute (neighbourhood) extensor coincide.

If $X \in A[N] E, Z$ is a $G$-space from the class $\mathscr{G}$ and $\varphi$ is a $G$-map (which in this case means that $\varphi$ is constant along every orbit), then the extension $\hat{\varphi}$ can also be chosen to be a $G$-map. This follows from the closedness of $A / G$ in $Z / G$ (which, in turn follows by the closedness of $A$ in $Z$ ). In connection with this example we introduce some more general concepts.

DEFINITION 2.8. A $G$-space $X$ is called an equivariant absolute neighbourhood extensor, $X \in G$-ANE, if every partial $G$-map $Z \hookleftarrow A \stackrel{\varphi}{\rightarrow} X$, where $Z$ is a $G$-space from the class $\mathscr{G}$, can be extended to a $G$-map $\hat{\varphi}: U \rightarrow X$, defined on some $G$ neighbourhood $U \subset Z$ of $A$. If we can always take $U=Z$ then $X$ is called an equivariant absolute extensor, $X \in G-A E$.

DEFINITION 2.9. A $G$-space $X$ is called an approximate $G$-A[N]E-space, $X \in$ $G-A A[N] E$, if for every $G$-space $Z$ from the class $\mathscr{G}$, every fundamental subset $F$ of $Z$, and every covering $\omega \in \operatorname{cov}(X)$, the following holds: For every partial $G$-map $Z \hookleftarrow A \stackrel{\varphi}{\rightarrow} X$ there is an 'approximate' $G$-extension $\tilde{\varphi}: Z \rightarrow X$ [respectively $\tilde{\varphi}: U \rightarrow X$, where $U \subset Z$ is a $G$-neighbourhood of $A]$ such that the restrictions $\left.\varphi\right|_{A \cap F}$ and $\left.\tilde{\varphi}\right|_{A \cap F}$ are $\omega$-close, that is, $\left(\left.\varphi\right|_{A \cap \varphi},\left.\tilde{\varphi}\right|_{A \cap F}\right) \prec \omega$ (see [13]).

\section{Equivariant extensors for locally compact Lie groups}

For our purposes, the most important example of a proper $\mathrm{GL}(n)$-space is the space $C(n)$ of all convex bodies.

PROPOSITION 3.1. For every $n, C(n)$ is a proper $G L(n)$-space. 
PROOF. It suffices to prove that the following closed set

$$
C(r, R)=\left\{V \in C(n) \mid B^{n}(r) \subset V \subset B^{n}(R)\right\},
$$

where $B^{n}(a)$ denotes the closed ball with center at 0 and of radius $a$, is thin for every $0<r<R<\infty$, that is, that the set

$$
\mathscr{R}=((C(r, R), C(r, R))=\{g \in \mathrm{GL}(n) \mid g C(r, R) \cap C(r, R) \neq \emptyset\}
$$

is precompact.

Suppose not. Then for some sequence $g_{n}=\left\|g_{i j}^{n}\right\| \in \mathscr{R}$ and some indices $\left(i_{0}, j_{0}\right)$, one of following cases occur

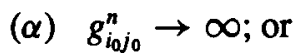

(B) $\operatorname{det}\left\|g_{i j}^{n}\right\| \rightarrow 0$.

Suppose that $g_{n} V_{n} \in C(r, R)$ for some $V_{n} \in C(r, R)$. Since the point $A$, for which only the $j_{0}$-th coordinate is equal to $r$, while all others are 0 , lies in $V_{n}$, it follows that $g_{n} A \in B^{n}(R)$. But the $i_{0}$-th coordinate of $g_{n} A$ is equal to $g_{i_{0} j_{0}}^{n} r$ and $g_{i_{0} j_{0}}^{n}$ does not converge to $\infty$. On the other hand,

$$
0<\operatorname{vol} B^{n}(r) \leq \operatorname{vol}\left(g_{n} V_{n}\right)
$$

and

$$
\operatorname{vol}\left(g_{n} V_{n}\right)=\operatorname{det}\left\|g_{i j}^{n}\right\| \text { vol } V_{n} \leq \operatorname{det}\left\|g_{i j}^{n}\right\| \operatorname{vol} B^{n}(R) .
$$

Therefore, det $\left\|g_{i j}^{n}\right\|$ does not converge to 0 .

The orthogonal group $O(n)$ is a maximal compact subgroup of GL(n). By Theorem 2.6 there exists a global $O(n)$-slice $f: C(n) \rightarrow \mathrm{GL}(n) / O(n)$.

PROPOSITION 3.2. Let $X$ be a proper G-ANE-space. Then

$(\gamma)$ For every $G$-neighbourhood U of the orbit $G(x)$, there exist a $G$-neighbourhood $V$ and a $G$-map $H: V \times[0,1] \rightarrow U$ such that $H_{0}=\operatorname{Id}, \operatorname{Im}\left(H_{1}\right) \subset G(x)$, and $\left.H_{t}\right|_{G(x)}=$ Id for all $t \in I$.

Proof. Consider in the proper $G$-space $X \times[0,1]$ the partial $G$-map

$$
X \times[0,1] \leftrightarrow X \times\{0\} \cup G(x) \times[0,1] \cup U_{1} \times\{1\} \stackrel{\varphi}{\rightarrow} X
$$

such that $\left.\varphi\right|_{X \times\{0\}}=\mathrm{Id},\left.\varphi\right|_{G(x) \times[0,1]}=\mathrm{Id}$, and $\left.\varphi\right|_{U_{1} \times\{1\}}$ is the existing retraction (provided by Theorem 2.6) $r: U_{1} \rightarrow G(x)$ of some $G$-neighbourhood $U_{1} \subset U$.

Let $\tilde{\varphi}: W \rightarrow X$ be any extension of $\varphi$ onto the $G$-neighbourhood $W$, which contains a $G$-neighbourhood of the type $V \times I \supset G(x)$. We get the desired map $H$ by restricting $\hat{\varphi}$ onto $V \times I$. 
The following theorem of Abels $[2,4.4]$ allows us to reduce the studying of noncompact group actions to compact ones.

THEOREM 3.3. For every $X \in \mathscr{G}, X \in G \cdot A[N] E$ if and only if $X \in L-A[N] E$ for every compact subgroup $L<G$.

THEOREM 3.4. For every $n, C(n)$ is $a$ GL( $(n)-A E$ space.

By Theorem 3.3, $C(n) \in \mathrm{GL}(n)-A E$ if and only if $C(n) \in L-A E$, for every compact subgroup $L<\operatorname{GL}(n)$. Another theorem of Abels [2, 4.2] asserts that every locally convex complete topological vector $G$-space is $G-A E$, for every compact group $G$. Let us apply the argument from this paper to prove that $C(n) \in L-A E$.

Since $C(n)$ is convex (with respect to the Minkowski linear combination of convex bodies), Dugunji's theorem implies that $C(n) \in A E$. Therefore every partial $L$-map $Z \hookleftarrow A \stackrel{f}{\rightarrow} C(n)$ can be continuously extended over $Z, F: Z \rightarrow C(n)$. Now define

$$
\hat{F}(z)=\int_{L} g^{-1} \cdot F(g z) \partial \mu,
$$

where $\partial \mu$ is the normalized Haar measure and $\int_{L}$ means the integral of the set-valued mapping [9]:

$$
\Phi_{z}: L \leadsto \mathbb{R}^{n}, \Phi_{z}(g)=g^{-1} \cdot F(g z) \subset \mathbb{R}^{n} .
$$

On account of the continuous dependence $\Phi_{z}(g)$ on $z$ and $g$, the convexity and the closeness of its images, $\hat{F}$ is a continuous map with closed convex values [9]. It is easy to see that $\hat{F}$ is an $L$-map from $Z$ into $C(n)$ and that $\hat{F} \mid A=f$.

Let $(X, d)$ be a metric $\mathrm{G}$-space of diameter 1 from $\mathscr{G}$. Then we can introduce a metric on the cone $\operatorname{Con} X=X \times[0,1] / X \times\{0\}$ as follows:

$$
\rho\left((x, t),\left(x^{\prime}, t^{\prime}\right)\right)=\sqrt{t^{2}+\left(t^{\prime}\right)^{2}-2 t t^{\prime} \cos \gamma}, \quad \text { where } \cos \gamma=\frac{2-d^{2}\left(x, x^{\prime}\right)}{2} .
$$

It is easy to see that (Con $X, \rho$ ) is a metric G-space (the group $G$ acts along $X$ ) and the natural embedding $X \mapsto X \times\{1\} \hookrightarrow \operatorname{Con} X$ is an isometry, while Con $X$ is not a proper space.

PROPOSITION 3.5. If a metric G-space $X$ is a G-ANE space, then Con $X$ is a G-AE space.

ProOF. Suppose that a proper $G$-space $Z \in \mathscr{G}$ and a partial G-map $Z \hookleftarrow A \stackrel{\varphi}{\rightarrow}$ Con $X$ are given. Let $A_{0}=\varphi^{-1}(*) \subset A$, where $(*)$ is the vertex of Con $X$. Then 
for every $a \in A \backslash A_{0}, \varphi(a)$ can be represented in the form $\left(\varphi_{1}(a), \varphi_{2}(a)\right)$, where $\varphi_{1}: A \backslash A_{0} \rightarrow X$ is a continuous $G$-map and $\varphi_{2}: A \rightarrow[0,1]$ is a continuous function, constant on the orbits and such that $\varphi_{2}\left(A \backslash A_{0}\right) \subset(0,1]$ and $\varphi_{2}\left(A_{0}\right)=0$.

Since $X \in G-A N E$, the map $\varphi_{1}(a)$ can be extended to a G-map $\psi: U \rightarrow X$, defined on an open subset $U$ of $Z / G, Z \backslash A_{0} \supset U \supset A \backslash A_{0}$. Since the orbit space $Z / G$ is metrizable, there exists a continuous function $\xi: Z \rightarrow[0,1]$, constant on orbits, such that $\left.\xi\right|_{A}=\varphi_{2}$ and $\left.\xi\right|_{Z \backslash U}=0$ by the Urysohn theorem. The desired extension $\hat{\varphi}: Z \rightarrow \operatorname{Con} X$ of the $G$-map $\varphi$ is then defined by the formula:

$$
\hat{\varphi}= \begin{cases}(\psi(z), \xi(z)) & z \in U ; \\ (*) & z \notin U .\end{cases}
$$

PROPOSITION 3.6. Let $H$ be a compact subgroup of the locally compact Lie group $G$. Then $G / H$ is a $G$-ANE-space.

PROOF. Every compact subgroup $H<G$ smoothly acts on the differentiable manifold $G / H$. By $[21,1.6 .6], G \in H-A N E$. By Theorem 3.3, $G \in G-A N E$.

It is convenient to reduce the studying of the equivariant extensors to the corresponding easier problem for approximate equivariant extensors. For example, if some class $\mathscr{B}$ of $G$-spaces is invariant under the product on the semiopen segment $J=[0,1)$, then $\mathscr{B}$ is contained in the class $G-A[N] E$ if and only if $\mathscr{B}$ is contained in the class of the approximate $G-A[N] E$.

THEOREM 3.7. Suppose that the product $X \times J$ of a metric $G$-space $X$ and $J=$ $[0,1)$ is a G-AANE-space. Then $X$ is a G-ANE-space.

For the trivial group $G$ this is a well-known fact, which follows from [12] and [18].

PROOF OF THEOREM 3.7. First, we consider any (not necessarily locally finite) covering $\omega \in \operatorname{cov}(X \times J)$ adjoining to the subset $X \times\{1\}$ of $X \times[0,1]$. The latter means by definition that:

( $\delta$ ) For every neighbourhood $U(x, 1)$ of the point $(x, 1) \in X \times\{1\}$ in $X \times[0,1]$, there exists a smaller neighbourhood $V(x, 1)$ such that $W \subset U(x, 1)$, for every $W \in \omega$ such that $W \cap V(x, 1) \neq \emptyset$.

Let $F$ be a fundamental set of $Z$ (see Proposition 2.3). Then $F \times J$ is a fundamental set of $Z \times J$. After these preliminaries, we begin the extending of the partial $G$-map $Z \hookleftarrow A \stackrel{\varphi}{\rightarrow} X$. Recall that $X \times J \in G-A A[N] E$ and construct for the other partial $G$-map

$$
Z \times J \hookleftarrow A \times J \stackrel{\psi=\varphi \times I \mathrm{Id},}{\longrightarrow} X \times J
$$


a $G$-map $\tilde{\psi}: Z \times J \rightarrow X \times J$ [respectively $\tilde{\psi}: U \rightarrow X \times J$ ] such that

$$
\left(\left.\varphi\right|_{(A \cap F) \times J},\left.\tilde{\psi}\right|_{(A \cap F \times J)}\right) \prec \omega .
$$

We give all details of the proof only for the case when $X \times J \in G-A A E$. The case when $X \times J \in G-A A N E$ is dealt with similarly. Extending $\tilde{\psi}$ over $A \times\{1\}$ by the formula $\tilde{\psi}(a, 1)=(\varphi(a), 1)$, we obtain a $G$-map (which we denote by the same letter) $\tilde{\psi}: Z \times J \cup A \times[0,1] \rightarrow X \times J$, the restrictions of which onto the closed $G$-set $A \times[0,1]$ and the open $G$-set $Z \times J$ are continuous. Now we apply the following lemma.

LEMMA 3.8. Suppose that a G-map $f: H \cup E \rightarrow Y$ is defined on the union $H \cup E$ of a closed $G$-space $H \in \mathscr{G}$ and open $G$-subset $E$ of a proper $G$-space $T \in \mathscr{G}$, such that $\left.f\right|_{H}$ and $\left.f\right|_{E}$ are continuous. Then there exists a closed $G$-subspace $K \subset T$ such that $H \subset K \subset H \cup E, H \cap U \subset \operatorname{Int}(K)$ and $\left.f\right|_{K}$ is a continuous G-map.

Apply Lemma 3.8 for $T=Z \times[0,1], H=A \times[0,1], E=Z \times J$ and $f=\tilde{\psi}$. We get a closed $G$-subset $L$ of $Z \times[0,1]$ such that $A \times[0,1] \subset L, A \times[0,1) \subset \operatorname{Int} L$ and $\left.\tilde{\psi}\right|_{L}$ is a continuous $G$-map.

Next, we construct a decreasing sequence $L=U_{1} \supset \mathrm{Cl} U_{2} \supset \cdots$ of open $G$ neighbourhoods of the set $A$ and a monotone sequence of numbers $0=t_{1}<t_{2}<\cdots$, such that $\lim _{i \rightarrow \infty} t_{i}=1$ and $U_{k} \times\left[0, t_{k}\right] \subset L$.

Let $\xi: Z \rightarrow[0,1]$ be a continuous real-valued function, constant on the orbits and such that $\xi\left(U_{1} \backslash U_{2}\right)=0, \xi\left(U_{i} \backslash U_{i+1}\right) \subset\left[t_{i-1}, t_{i}\right]$ for every $i \geq 2$, and $\xi(A)=1$. Clearly, the graph GR $=\{(z, \xi(z)) \mid z \in Z\}$ of $f$ lies in $L$ and the restriction of $\tilde{\psi}$ onto GR is a continuous $G$-map. The desired extension is now given by the formula:

$$
\hat{\varphi}(z) \text { is the projection of } \tilde{\psi}(z, \xi(z)) \in X \times[0,1] \text { onto } X .
$$

Using diam $W_{n} \rightarrow 0, W_{n} \in \omega$, whenever $\operatorname{dist}\left((x, 1), W_{n}\right) \rightarrow 0$ for some point $(x, 1) \in X \times\{1\}$, it is easy to check the continuity of $\hat{\varphi}$.

\section{Orbit spaces of equivariant absolute extensors}

This section is dedicated to a proof of the following result.

THEOREM 4.1. Let $G$ be a locally compact Lie group and $X$ a proper $G-A[N] E$ from $\mathscr{G}$. Then the orbit space $X / G$ is an absolute [neighbourhood] extensor.

Since $C(n)$ is a proper GL(n)-space from $\mathscr{G}$ which is an equivariant absolute extensor, we obtain as an immediate corollary of Theorem 4.1 that for every closed 
subgroup $H<\mathrm{GL}(n)$, the orbit space $C(n) / H$ belongs to the class of absolute extensors.

We begin with the following embedding theorem.

PROPOSITION 4.2. Let $X \in \mathscr{G}$. Then there exist a countable number of finitedimensional $G$-ANE-spaces $R_{n m}\left(n, m \in \mathbb{Z}^{+}\right)$, from the class $\mathscr{G}$, and a topological G-embedding $i: X \hookrightarrow \prod_{n, m}^{\infty}$ Con $R_{n, m}$.

Let $X$ be equipped by the invariant metric (see Proposition $2.7(\mathrm{~g})$ ). For every point $x$ and every $\varepsilon>0$, we fix a G-map $\varphi_{x \varepsilon}: X \rightarrow \operatorname{Con}(G(x))$ satisfying the properties of the following proposition.

PROPOSITION 4.3. Let $X \in \mathscr{G}$. Then for every point $x \in X$ and every $\varepsilon>0$, there exists a G-map $\varphi: X \rightarrow \operatorname{Con}(G(x))$ with $\varphi(x)=x$, such that

(5) $\operatorname{diam} \varphi^{-1}((V \cdot x) \times(0,1])<\varepsilon$, for some neighbourhood $V$ of the stabilizer $G_{x}$ in $G$.

PROOF. Let $r: U(x) \rightarrow G(x)$ be a G-retraction. We may assume that not only does the $G_{x}-\operatorname{kernel} r^{-1}(x)$ have diameter less than $\varepsilon$, but also diam $\left(V \cdot r^{-1}(x)\right)<\varepsilon$, for some neighbourhood $V$ of the compact stabilizer $G_{x}$. This is possible by Theorem 2.5 and the following lemma.

LEMMA 4.4. For every neighbourhood $O(x) \subset X$, there exists a smaller neighbourhood $O_{1}(x)$ such that

(6) $G_{x} \cap \operatorname{cl}\left\{g \mid g O_{1}(x) \backslash O(x) \neq \emptyset\right\}=\emptyset$; and

(7) $G \cdot O_{1}(x) \cap r^{-1}(x) \subset O(x)$.

The desired $G$-map of $X$ is then given by the formula:

$$
\varphi\left(x^{\prime}\right)= \begin{cases}\left(r\left(x^{\prime}\right), \xi\left(x^{\prime}\right)\right) & x^{\prime} \in U(x) ; \\ (*) & x^{\prime} \notin U(x) .\end{cases}
$$

Here, the function $\xi: X \rightarrow[0,1]$ is constant on orbits, $\xi(x)=1$ and $\xi(X \backslash U(x))=0$.

Since by hypothesis $X / G$ is metrizable, there exists a $\sigma$-disjoint basis $\mathscr{B}=$ $\left\{W_{\mu}\right\}_{\mu \in M}$ of open subsets, such that $\mathscr{B}=\bigsqcup \mathscr{B}_{n}$, where $\mathscr{B}_{n}=\left\{W_{\mu}\right\}_{\mu \in M_{n} \subset M}$ is a disjoint family and $\bigsqcup_{n=1}^{\infty} M_{n}=M$.

DEFINITION 4.5. A pair $v=\left(\mu_{1}, \mu_{2}\right) \in M \times M$ of indices is said to be canonical, if

(8) $W_{\mu_{1}} \Subset W_{\mu_{2}}$ (that is, $\bar{W}_{\mu_{1}} \subset W_{\mu_{2}}$ ); and

there exist $x \in X$ and $\varepsilon>0$ such that: 
(9) $x \in \pi^{-1} W_{\mu_{1}} \subset V_{x \varepsilon}$ and $U_{x \varepsilon} \subset \pi^{-1} W_{\mu_{1}}$, where

$$
V_{x \varepsilon}=\varphi_{x \varepsilon}^{-1}\left(G_{x} \times(1 / 2,1]\right) \quad \text { and } U_{x \varepsilon}=\varphi_{x \varepsilon}^{-1}\left(G_{x} \times(0,1]\right) \text {, }
$$

and $\pi: X \rightarrow X / G$ is the orbit projection.

We denote the set of all canonical pairs by $K \subset M \times M$.

PROPOSITION 4.6. There exists a correspondence $v \in K \longmapsto\left(x_{v}, \varepsilon_{v}\right) \in X \times \mathbb{R}^{+}$ such that $\left(x_{v}, \varepsilon_{v}\right)$ satisfies $(9)$ and

(10) For every closed subset $F \subset X$ and $x \notin F$ there exists a canonical pair $v \in K$ with $\varphi_{x_{v} \varepsilon_{v}}(x) \notin \varphi_{x_{v} \varepsilon_{v}}(F)$ (that is, $\varphi_{x_{v} \varepsilon_{v}}$ separates the point $x$ from the closed subset $F$ ).

PROOF. Let

$$
i(v)=\inf \{\varepsilon>0 \mid(x, \varepsilon) \text { satisfies (9) for some point } x \in X\} .
$$

It is evident that $i(\nu)>0$. Therefore, every $v \in K$ yields a pair $\left(x_{v}, \varepsilon_{v}\right)$ possessing (9) and such that

(11) $\varepsilon_{v}<2 i(v)$.

Let $4 a=\rho(x, F)$. Since $\mathscr{B}$ is a basis, there exist $\nu=\left(\mu_{1}, \mu_{2}\right) \in K$ and $\varepsilon<a$ such that

$$
x \in \pi^{-1} W_{\mu_{1}} \subset V_{x \varepsilon} \subset U_{x \varepsilon} \subset \pi^{-1} W_{\mu_{1}} .
$$

It follows from (11) that $\varepsilon_{v}<2 a$.

Let us prove that $v$ is a desired pair. Suppose that a neighbourhood $V$ of $G_{x_{v}}$ satisfies the hypotheses of Proposition 4.3:

$$
\operatorname{diam} \varphi_{x_{v} \varepsilon_{v}}^{-1}\left(\left(V x_{v}\right) \times(0,1]\right)<\varepsilon \nu<2 a .
$$

Since $x \in V_{x_{v} \varepsilon_{v}}$, it follows that $\varphi_{x_{v} \varepsilon_{v}}(x)=\left(g x_{v}, t\right), t>1 / 2$.

Pick a neighbourhood $W=g V g^{-1}$ of $e \in G$. Then

$$
\varphi_{x_{\nu} \varepsilon_{\nu}}(x) \in\left(W \cdot g x_{\mu}\right) \times(1 / 2,1]
$$

and

$$
\begin{aligned}
& A=\varphi_{x_{v} \varepsilon_{v}}^{-1}\left(W \cdot g x_{\mu} \times(1 / 2,1]\right) \\
& \quad \subset \varphi_{x_{v} \varepsilon_{\nu}}^{-1}\left(g \cdot V \cdot x_{\mu} \times(1 / 2,1]\right)=g \cdot \varphi_{x_{v} \varepsilon_{v}}^{-1}\left(V \cdot x_{\mu} \times(1 / 2,1]\right) .
\end{aligned}
$$

By the invariance of the metric, the latter set has diameter smaller than $2 a$, hence the diameter of the open neighbourhood $A$ of $x$ is also less than $2 a$. As a consequence, it follows that $A \cap F=\emptyset$ and $\varphi_{x_{v} \varepsilon_{v}}(x) \notin \varphi_{x_{v} \varepsilon_{v}}(F)$. 
PROOF OF PROPOSITION 4.2. Let us introduce a countable family of spaces:

$$
R_{n m}=\coprod\left\{G\left(x_{v}\right) \mid v=\left(\mu_{1}, \mu_{2}\right) \in K, \mu_{1} \in \mathscr{B}_{n}, \mu_{2} \in \mathscr{B}_{m}\right\} .
$$

Since $G\left(x_{v}\right) \in G-A N E, R_{n m}$ is also a $G-A N E$. Since $\mathscr{B}_{m}$ is a disjoint family and

$$
\left.\varphi_{x_{\nu} \varepsilon_{\nu}}\right|_{\left(X \backslash \pi^{-1} W_{\mu_{2}}\right)}=(*) \in \operatorname{Con}\left(G\left(x_{\nu}\right)\right),
$$

we obtain that

$$
\psi_{n m}: X \rightarrow \operatorname{Con} R_{n m},\left.\quad \psi_{n m}\right|_{\pi^{-1}} W_{\mu_{2}}=\varphi_{x_{v} \varepsilon_{\nu}},\left.\quad \psi_{n m}\right|_{X \backslash \cup \pi^{-1} W_{\mu_{2}}}=(*)
$$

is a well-defined $G$-map. Since $\left\{\psi_{n m}\right\}$ separates points from closed subsets, the diagonal product

$$
\Delta \psi_{n m}: X \rightarrow \prod_{n, m} \operatorname{Con} R_{n m}
$$

is a topological $G$-embedding.

Proposition 4.7. Suppose that a $G$-space $H$ is the limit of the inverse spectrum $\left\{H_{1} \stackrel{q_{1}}{\leftarrow} H_{2} \stackrel{q_{2}}{\leftarrow} H_{3} \leftarrow \cdots\right\}$ of $G$-spaces $H_{i}$ and $G$-maps $q_{i}$, and that

(12) The stabilizer $G_{h}$ of any point $h \in H_{i} \backslash H_{i}^{G}$ is compact.

Then the orbit spaces $H / G$ and $\lim \left\{H_{1} / G \stackrel{\tilde{q}_{1}}{\leftarrow} H_{2} / G \stackrel{\tilde{q}_{2}}{\leftarrow} H_{3} / G \leftarrow \cdots\right\}$ are homeomorphic.

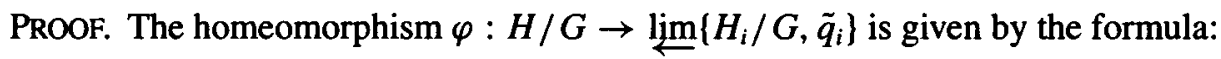

$$
\varphi([h])=\left(\left[h_{1}\right],\left[h_{2}\right], \ldots\right), \text { where } h=\left(h_{i}\right) \in H .
$$

It is easy to verify that $\varphi$ is continuous and surjective. We shall thus only verify that $\varphi$ is injective. Assume that $[h] \neq[e]$, where $h=\left(h_{i}\right), e=\left(e_{i}\right) \in H$ and let us show that then $\varphi([h]) \neq \varphi([e])$. It suffices to prove the following lemma.

LEMMA 4.8. There exists an integer $i$ such that $e_{i} \notin G\left(h_{i}\right)$.

Proof. If $e, h \in H^{G}$, then $e_{i} \neq h_{i}=G \cdot h_{i}$, for some $i$. So we may assume that $h \notin H^{G}$, that is, $G_{h}=\cap G_{h_{i}} \neq G$. By (12) and inclusion $G_{h_{i+1}} \subset G_{h_{i}}$, almost all $G_{h_{i}}$ 's differ from $G$ and almost all $G_{h_{i}}$ are compact.

Suppose to the contrary, that $e_{i}=g_{i} h_{i}, g_{i} \in G$ for every $i$. It is easy to show that then

$$
e_{k}=g_{k} h_{k}=g_{k+1} h_{k}=\cdots=g_{l} h_{k}
$$


for every $k \leq l$. Therefore, $g_{l} \in g_{k} \cdot G_{h_{k}}$, for every $k \leq l$.

Since the stabilizer $G_{h_{m}}$ is compact for some $m$, it follows that the sequence $\left\{g_{l}\right\}_{l \geq m} \subset g_{m} \cdot G_{h_{m}}$ converges to $g_{0} \in g_{m} \cdot G_{h_{m}}$. Analogously, one can show that $g_{0} \in g_{p} \cdot G_{h_{p}}$, for all $p \geq m$. Consequently, $g_{0} h_{p}=g_{p} h_{p}=e_{p}$, for all $p \geq m$, that is, $e=g_{0} h$. Contradiction.

PROOF OF THEOREM 4.1. Using the hypotheses, let us fix a topological $G$-embedding (Proposition 4.2):

$$
i: X \hookrightarrow \prod_{n, m} \operatorname{Con} R_{n m}=D
$$

and a closed topological embedding $j: X / G \hookrightarrow L$ of the orbit space $X / G$ into a linear normed space $L$. It is obvious that

$$
i \times(j \circ \pi)=e: X \hookrightarrow L \times D
$$

is a closed topological $G$-embedding. Since the image $e(X)$ does not contain points with a noncompact stabilizer, $e(X)$ does not intersect the closed set $L \times\{*\}$, where $\{*\}$ is the product of the vertices of the cone-factors of $D$. Therefore, $e(X)$ lies in the proper open $G$-space $U^{\prime}=L \times(D \backslash\{*\})$.

Since $L \times D \in \mathrm{G}-A E$, it follows that $U^{\prime} \in \mathrm{G}-A N E$. Since $X \in \mathrm{G}-A N E$, there exists a $G$-retraction $r: U \rightarrow X$ of some $G$-neighbourhood $U, e(X) \subset U \subset U$. Hence, $\tilde{r}: U / G \rightarrow X / G$ is a retraction and the inclusion $X / G \in A N E$ is reduced to another inclusion $U / G \in A N E$.

If we now prove that $D / G \in A E$, then $(L \times D) / G=L \times(D / G) \in A E$, and therefore, $U / G \in A N E$ as an open subset of the orbit space. To complete the proof of the theorem, it thus remains to verify that $D / G \in A E$.

Let us introduce the following notations: $D_{p}=\prod_{n+m \leq r} \operatorname{Con} R_{n m}$ and $q_{r}: D_{r+1} \rightarrow$ $D_{r}$ is a projection. Since $R_{n m}$ is metrized by a complete invariant metric, it follows that Con $R_{n m}$ and $D_{r}$ are also metrized by a complete invariant metric. Thus, the orbit space $D_{r} / G$ is also metrized by a complete metric. It follows from $D_{r} \in G-A E$ and Proposition 3.2 that $D_{r} / G \in \mathrm{LC} \cap \mathrm{C}$. Due to its countable-dimensionality and the Haver theorem [15] we obtain that $D_{r} / G \in A E$.

Since Con $R_{r+1} \in A E$, the projection $q_{m}$ is a fiberwise $G$-contractible map, that is, there exist fiberwise $G$-maps $s: D_{r} \rightarrow D_{r+1}, q_{r} \circ s=$ Id and $H: D_{r+1} \times[0,1] \rightarrow$ $D_{r+1}, q_{r} \circ H=q_{r}$, such that $H_{0}=\operatorname{Id}$ and $\operatorname{Im}\left(H_{1}\right)=\operatorname{Im}(s)$. Passing to the orbit spaces we obtain fiberwise contractible maps $\tilde{q}_{r}: D_{r+1} / G \rightarrow D_{r} / G$, that is, $\tilde{q}_{r}$ is a fine homotopy equivalence. Since all the conditions of Curtis's theorem [11] are satisfied, we conclude that $\lim \left\{D_{i} / G, q_{i}\right\}$ is an $A E$. But by Proposition 4.7 this inverse limit coincides with the orbit space $D / G$. 


\section{Proof of Theorem 1.2}

By Theorem 2.6 and Proposition 3.1, there exists a GL(n)-retraction $r: C(n) \rightarrow$ $\mathrm{GL}(n) / O(n)=\mathbb{E}$, which is nevertheless unacceptable for us because of its nonconstructibility. Another geometric GL( $n$ )-retraction, generated by the Löwner ellipsoid, will be more convenient.

THEOREM 5.1 (see [17]). For every convex body $V \in C(n)$, there exists a unique ellipsoid $E_{V} \in C(n)$, which contains $V$ and has the minimal Euclidean volume.

The GL(n)-invariance of $E_{V}$ (that is, $E_{A V}=A E_{V}$ for all $A \in \mathrm{GL}(N)$ ) then follows by minimality of the volume. A continuous dependence $E_{V}$ on $V$ with respect to the Hausdorff metric was proved in [5]. Therefore, $\mathscr{L}: C(n) \rightarrow \mathfrak{E}, \mathscr{L}(V)=E_{V}$, is a $\mathrm{GL}(n)$-retraction of $C(n)$ onto the ellipsoid orbit $\mathbb{E}$ ( $\mathscr{L}$ is called the Löwner retraction).

Since the symmetry group $\operatorname{Sym}_{B^{n}}$ of $B^{n}$ is $O(n)$, the $O(n)$-slice $L(n)=\mathscr{L}^{-1}\left(B^{n}\right)$ is an $O(n)$-space. In other words, $L(n)$ consists of all bodies $V \in C(n)$ whose minimal Löwner ellipsoid coincides with $B^{n}$. The orbit space $Q(n)=C(n) / \mathrm{GL}(n)$ is homeomorphic to $L(n) / O(n)$. Therefore, by Theorem 4.1,

$$
L(n) / O(n)=Q(n) \in A E \text { and } Q_{b}=Q(n) \backslash\{\text { Eucl. }\}=L_{\delta}(n) / O(n) \in A N E \text {, }
$$

where $L_{\delta}=L(n) \backslash\left\{B^{n}\right\}$, and so Theorem 1.2 is reduced to the following:

THEOREM 5.2. $Q_{\delta}(2)=L_{\delta}(2) / O(2)$ is a Hilbert cube manifold.

We prove Theorem 5.2 in three main steps which are carefully outlined below.

\section{Step 1. Reduction of Theorem 5.2 to Proposition 5.3 and the Torunczyk characterization for $Q$-manifolds}

PROPOSITION 5.3. For every integer $n \geq 2$ and every $\delta>0$, there exist $O(n)$-maps $f_{i}: L_{\mathscr{E}}(n) \rightarrow L_{\mathscr{E}}(n), i \in\{1,2\}$, such that

(1) $f_{i}$ and $\operatorname{Id}_{L_{\delta}(n)}$ are $\delta$-close; and

(2) if $n=2$ then $\operatorname{Im} f_{1} \cap \operatorname{Im} f_{2}=\emptyset$.

PROOF OF THEOREM 5.2. According to the Toruńczyk characterization criterion [19], in order to prove Theorem 5.2, it suffices to check that for every $\varepsilon>0$ and for all pairs of maps $\varphi_{i}: I^{\infty} \rightarrow Q_{\delta}(n), i \in\{1,2\}$, there are continuous maps $g_{i}: I^{\infty} \rightarrow Q_{\delta}(n), \varepsilon$-close to $\varphi_{i}, i \in\{1,2\}$, such that if $n=2$ then $\operatorname{Im} g_{1} \cap \operatorname{Im} g_{2}=\emptyset$. 
Since $F=\cup \operatorname{Im} \varphi_{i}$ and $F_{1}=\pi^{-1}(F)$ are compact (here $\pi: L_{\delta}(n) \rightarrow L_{\delta}(n) / O(n)$ is the orbit projection), there exists $\delta>0$ such that $\operatorname{dist}(\pi(a), \pi(b))<\varepsilon$, for every $a, b \in F_{1}$, with $\operatorname{dist}(a, b)<\delta$.

By Proposition 5.3 for every $n \geq 2$, there are $O(n)$-maps $f_{i}: L_{\delta}(n) \rightarrow L_{\varepsilon}(n)$, $i \in\{1,2\}$, satisfying (1) for $\delta>0$ and (2) for $n=2$. The induced maps $\tilde{f_{i}}$ of the orbit spaces, $i \in\{1,2\}$, have the following properties for $n=2$ :

$$
\rho\left(\left.\tilde{f_{i}}\right|_{F}, \operatorname{Id}_{F}\right)<\varepsilon \text { and } \cap \operatorname{Im} \tilde{f_{i}}=\emptyset .
$$

Finally, the desired maps $g_{i}: I^{\infty} \rightarrow Q_{\delta}(2), i \in\{1,2\}$, are defined by the formula $g_{i}=\tilde{f_{i}} \circ \varphi_{i}$.

\section{Step 2. Construction of $f_{1}$}

Let us consider so-called contact map $\alpha: L(n) \rightarrow \exp \left(S^{n-1}\right)$, defined by $\alpha(V)=$ $V \cap S^{n-1}$. The following lemma, whose routine verification is omitted, records several basic properties of $\alpha$.

LEMMA 5.4. (3) $\alpha$ preserves the action of $O(n), \alpha(A \cdot V)=A \cdot \alpha(V)$, for every $A \in O(n)$

(4) $\alpha(V) \neq \emptyset$, for every $V \in L(n)$;

(5) $\alpha(V)$ is a central symmetric subset of $S^{n-1}$; and

(6) $\alpha(V)=S^{n-1}$ if and only if $V=B^{n}$.

LEMMA 5.5. (7) Let $V \subseteq W \subseteq B^{n}$, where $V \in L(n)$ and $W \in C(n)$. Then $W \in L(n)$.

(8) For every subset $A \subseteq B^{n}, \alpha(\operatorname{Conv}(A))=\operatorname{Conv}(A) \cap S^{n-1}=A \cap S^{n-1}$.

PROOF. (7) The minimal Löwner ellipsoid for $W$ and $V$ coincides with $B^{n}$. Hence $W \in L(n)$.

In order to prove (8), it suffices to observe that every point $s \in \operatorname{Conv}(A) \cap S^{n-1}$ is an extreme point of $B^{n}$ and therefore is also an extreme point of $\operatorname{Conv}(A) \subseteq B^{n}$. But all extreme points of $\operatorname{Conv}(A)$ are contained in $A$. Therefore $s \in A$.

Unfortunately, the contact map $\alpha$ is discontinuous. The following reasoning compensates for this unpleasant moment. Let us denote by $\widehat{x 0 y}$ the nonoriented angle between the rays $[0 x)$ and $\left[0 y\right.$ ), where $x, y \in B^{n}$ and $x, y \neq 0$. Next, we introduce a version of the closed $\varepsilon$-neighbourhood of a set, which will be convenient for us. Let $\varepsilon>0$ and $V \in L(n)$. By $V_{\varepsilon}$ we denote

$V \cup\left\{x \in B^{n} \backslash\{0\} \mid\right.$ there exists $y \in V$ with $\|x\|=\|y\|$ and $\left.\widehat{x 0 y} \leq \varepsilon\right\}$. 
It is clear that $V_{\varepsilon}$ preserves the action of $O(n):(g \cdot V)_{\varepsilon}=g \cdot V_{\varepsilon}$, for every $g \in$ $O(n), V \in L_{\varepsilon}(n)$. The compactness of $V$ implies that $V_{\varepsilon}$ is compact; the inequality $\|x-y\|<\widehat{x 0 y}$, for every $\|x\|=\|y\|$, implies that

(9) $V \subseteq V_{\varepsilon} \subseteq \bar{N}(V ; \varepsilon)$, where $\bar{N}(V ; \varepsilon)$ is a closed $\varepsilon$-neighbourhood of $V$ in $B^{n}$.

We need $V_{\varepsilon}$ to be continuously dependent on $V$ and $\varepsilon$.

PROPOSITION 5.6. Let $\varepsilon_{k} \rightarrow \varepsilon>0$ and $V_{k} \in L(n) \rightarrow V$. Then $\left(V_{k}\right)_{\varepsilon_{k}} \rightarrow V_{\varepsilon}$.

PROOF. Let $R_{k}=\left(V_{k}\right)_{\varepsilon_{k}}$ and $R=V_{\varepsilon}$. Suppose that the assertion of the proposition is false, that is, that $R_{k} \nrightarrow R$. Then there exist $\alpha>0$ and a sequence $k_{i} \rightarrow \infty$ such that

(10) $x_{0} \notin N\left(R_{k_{i}} ; \alpha\right)$, for some $x_{0} \in R$; or

(11) there exists $x_{i} \in R_{k_{i}}, i \geq 1$, with $x_{i} \notin N(R ; \alpha)$.

In the first case, $\widehat{x_{0} 0 y_{0}} \leq \varepsilon$, for some $y_{0} \in V$, with $\left\|y_{0}\right\|=\left\|x_{0}\right\|$. Since $V_{k} \rightarrow V$, there exists a sequence $y_{k} \in V_{k} \rightarrow y_{0}$. It is easy to see that there exists a sequence $x_{k} \in B^{n} \rightarrow x_{0}, x_{k} 0 y_{k} \leq \varepsilon_{k},\left\|x_{k}\right\|=\left\|y_{k}\right\|$. It means that $x_{k} \in\left(V_{k}\right)_{\varepsilon_{k}}=R_{k}$, for every $k$ and the limit point $x_{0}$ of $\left\{x_{k}\right\}$ belongs to $N\left(R_{k_{i}} ; \alpha\right)$, for some $k_{i}$. This contradicts (10).

In the second case, there exists a sequence $\left\{y_{i} \in V_{k_{i}}\right\}$ such that $\left\|y_{i}\right\|=\left\|x_{i}\right\|$ and $\widehat{y_{i} 0 x_{i}} \leq \varepsilon_{k_{i}}$. By compactness of $B^{n}$, we can suppose that there exist the limits $y_{i} \rightarrow y \in V$ and $x_{i} \rightarrow x \in B^{n}$. Then $\|y\|=\|x\|$ and $\widehat{x 0 y} \leq \varepsilon$. Therefore, $x \in V_{\varepsilon}=R$. This contradicts the fact that $x_{i} \notin N(R ; \alpha)$.

Consider the following set-valued map:

$$
F: L_{\varepsilon}(n) \rightsquigarrow \mathbb{R}^{+}, \quad F(V) \stackrel{\text { def }}{=}\left\{t>0 \mid B^{n} \backslash N(V ; t) \neq \emptyset\right\},
$$

where $N(V ; t)$ is the open $t$-neighbourhood of $V$ in $B^{n}$.

Since $N(V ; t)$ is a continuous set-valued map from $L_{\varepsilon}(n) \times \mathbb{R}^{+}$into $B^{n}$ (in the Hausdorff metric) and $B^{n} \backslash V \neq \emptyset$, the map $F$ is lower semicontinuous and has domain $L_{\varepsilon}(n)$. Let us consider the function $f: \operatorname{Graph}(F) \rightarrow \mathbb{R}^{+}$given by $f(V, t)=t$ and defined on the graph $F$. Then the function $g: L_{\varepsilon}(n) \rightarrow \mathbb{R}^{+}$, defined by

$$
g(V)=\sup \left\{t>0 \mid B^{n} \backslash N(V ; t) \neq \emptyset\right\}=\sup \{f(V, t) \mid(V, t) \in \operatorname{Graph}(F)\}
$$

is well defined and lower semi-continuous [9, page 48] (in set-valued analysis $g$ is called a marginal function [24]).

By the Dowker theorem [13], there exists a continuous function $\gamma: L_{\varepsilon}(n) \rightarrow$ $\mathbb{R}^{+}$with $\gamma(V)<\delta \cdot g(V), V \in L_{\varepsilon}(n)$. By Proposition 5.6, it is clear that $V_{\gamma(V)}$ continuously depends on $V \in L_{\varepsilon}(n)$. The desired continuous $O(n)$-map $f_{1}: L_{\varepsilon}(n) \rightarrow$ $L_{\varepsilon}(n)$ is defined by setting $f_{1}(V)=\operatorname{Conv}\left(V_{\gamma(V)}\right)$. By $(9), f_{1}$ and $\operatorname{Id}_{L_{\varepsilon}(n)}$ are $\delta$-close. 
Let dist $(v, w)$ be the spherical distance between $v, w \in S^{n-1}$ and $\bar{N}_{\text {sph }}(A ; R)$ be the closed $R$-neighbourhood of the subset $A \subset S^{n-1}$ with respect to the spherical distance. By Lemma 5.5(8),

$$
\alpha \circ f_{1}(V)=\operatorname{Conv}\left(V_{\gamma(V)}\right) \cap S^{n-1}=V_{\gamma(V)} \cap S^{n-1}=\bar{N}_{\mathrm{sph}}(V ; \gamma(V)) .
$$

The last equality means the boundary of $f_{1}(V)$ to contain an open (nonempty) subset $S^{n-1}$, for every $V \in L_{\delta}(n)$. The mapping $f_{2}$ will be constructed without such property and therefore $\operatorname{Im} f_{1} \cap \operatorname{Im} f_{2}=\emptyset$.

\section{Step 3. Construction of $f_{2}$}

THEOREM 5.7. For every $\sigma>0$, there exists an $O(n)$-mapping $F: L_{\mathscr{E}}(n) \rightarrow C(n)$ such that

(12) $\rho\left(F, \operatorname{Id}_{L_{\varepsilon}(n)}\right)<\sigma$; and

(13) for every $V \in L_{\mathscr{E}}(n), F(V)=\operatorname{Conv}\left(\sum_{i=1}^{m} \lambda_{i} D_{i}\right)$, where $D_{i}$ is an $H_{i}$-orbit, $H_{i}$ is a proper subgroup of $O(n)$ and $\sum_{i=1}^{m} \lambda_{i}=1, \lambda_{i} \geq 0$.

In connection with this theorem we formulate a geometric conjecture, which is trivially true in dimension 2 . If Conjecture 5.8 is valid then our proof of Theorem 1.2 immediately generalizes to arbitrary $n \geq 2$.

CONJECTURE 5.8. The body $\sum_{i=1}^{m} \lambda_{i} D_{i}$ (hence also $\left.\operatorname{Conv}\left(\sum_{i=1}^{m} \lambda_{i} D_{i}\right)\right)$ in the formula (13) 'essentially differs' from the ball, that is, its boundary does not contain open subsets of the sphere.

PrOOF. By the Palais theorem (Theorem 2.5) any orbit $O(n) V, V \in L_{\mathscr{B}}(n)$, allows an $O(n)$-retraction $r_{V}^{\prime}: \mathscr{U}_{V} \rightarrow O(n) V, r_{V}^{\prime}(V)=V$. Here we can assume that:

$$
\rho_{H}\left(W, r_{V}^{\prime}(W)\right)<\sigma / 2 \text {, for all } W \in \mathscr{U}_{V} \text {. }
$$

LEMMA 5.9. For every $\theta>0$ there exists a finite set $K \subset \mathrm{Bd} V$ such that:

(i) $W=\operatorname{Conv}\left(\mathrm{St}_{V} K\right)$ and $V$ have equal stabilizers; and

(ii) $\rho_{H}(V, W)<\theta$.

PROOF. It follows from the existence of slices that for some numbers $\theta>\theta_{1}>0$ from $\rho_{H}\left(V, V^{\prime}\right)<\theta$ and $\mathrm{St}_{V^{\prime}} \supseteq \mathrm{St}_{V}$, it always follows that $\mathrm{St}_{V^{\prime}}=\mathrm{St}_{V}$. Consider a discrete subset $K \subset \mathrm{Bd} V$ such that $\rho_{H}(V$, Conv $K)<\theta_{1}$. Then

$$
V \supseteq \operatorname{Conv}\left(\operatorname{St}_{V} K\right)=W \supseteq \operatorname{Conv} K
$$

and therefore $\rho_{H}(V, W)<\theta_{1}$. Next, it follows from $\mathrm{St}_{W}=\mathrm{St}_{\mathrm{Conn}_{\left(\mathrm{St}_{V} K\right)}}=\mathrm{St}_{\mathrm{St}_{V} K} \supseteq \mathrm{St}_{V}$ and $\rho(V, W)<\theta_{1}$ that $\mathrm{St}_{W}=\mathrm{St}_{V}$. 
For every $V \in L_{\mathscr{E}}(n)$, fix $V^{\prime}=\operatorname{Conv}\left(H K_{V}\right) \in C(n)$ such that $H=\mathrm{St}_{V}, K_{V} \subset$ $\operatorname{Bd} V,\left|K_{V}\right|<\infty$ and $\rho_{H}\left(V, V^{\prime}\right)<\sigma / 2$. Let us introduce the composition

$$
r=h_{V} \circ r^{\prime}: \mathscr{U}_{V} \rightarrow O(n) V \rightarrow O(n) V^{\prime},
$$

where $h_{V}(g V)=g V^{\prime}$ is an $O(n)$-homeomorphism.

If we get $V^{\prime}$ sufficiently close to $V$ then we obtain the following:

(15) $\operatorname{dist}\left(W, r_{V} W\right)<\sigma$, for every $W \in \mathscr{U}_{V}$.

We inscribe a locally finite cover $\left\{T_{\mu}\right\}$ into the open cover $\left\{\mathscr{U}_{V} / O(n)\right\}$ of the orbit space $L_{\delta}(n) / O(n)=Q_{\delta}(n)$. Let $T_{\mu} \subset \pi\left(U_{V_{\mu}}\right)$.

We now define the desired $O(n)$-map $F: L_{\delta}(n) \rightarrow C(n)$ as follows:

$$
F(W)=\sum_{\mu} \gamma_{\mu}(\pi W) \cdot r_{V_{\mu}}(W), \quad W \in L_{\delta}(n),
$$

where $\left\{\gamma_{\mu}(\cdot)\right\}$ is a continuous partition of unity, subordinate to the cover $\left\{T_{\mu}\right\}$.

We verify the conditions (12) and (13) of Theorem 5.7. Let $T_{1}, \ldots, T_{m} \in\left\{T_{\mu}\right\}$ be all the elements which contain $\pi W$ and let $T_{i} \subset \pi\left(U_{V_{i}}\right)$. It follows by (4) that $\rho_{H}\left(W, r_{V_{i}}(W)\right)<\sigma$, for all $i$. Then by convexity of the ball of radius $\sigma$ at $C(n)$ we have that $\operatorname{dist}(W, F W)<\sigma$. Thus (12) has been verified.

Condition (13) follows, since $H$ Conv $K$ is a union of a finite number of $H$-orbits for every proper subgroup $H<O(n)$ and finite $K$.

It is well known [2] that there exists a $O(n)$-retraction $R: C(n) \rightarrow L(n)$ which takes $C_{\delta}(n)$ into $L_{\delta}(n)$. But we need the following precise result which follows from geometric considerations:

THEOREM 5.10. There exists a continuous $O(n)$-retraction $R: C(n) \rightarrow L(n)$, such that $V$ and $R(V)$ are affinely equivalent, for every $V \in C(n)$.

PROOF. Let $L(V)$ be the Löwner ellipsoid, circumscribed around $V, g \in \mathrm{GL}(n)$, $g(L(V))=B^{n}$. As is well known, $g$ can be represented as $g=g_{2} \circ g_{1}$, where $g_{2} \in O(n)$ and $g_{1}$ is self-adjoint. Here $R(V)=g_{1}(V)$.

Since $L(n)$ is compact, for every $\delta>0$ there exists $\sigma>0, \sigma<\delta / 2$, such that for every $V \in L(n)$ and every $W \in C(n)$,

$$
\rho_{H}(V, W)<\sigma \Rightarrow \rho_{H}(W, R(W))<\delta / 2
$$

By Theorem 5.7 there is a mapping $F: L_{\mathscr{E}}(n) \rightarrow C(n)$ such that $\rho\left(F, \operatorname{Id}_{L_{\varepsilon}(n)}\right)<\sigma$. The desired $\operatorname{map} f_{2}$ is $R \circ F$. 
Indeed,

$$
\begin{aligned}
\rho_{H}\left(V, f_{2} V\right) & =\rho_{H}(V, R \circ F(V)) \\
& <\rho_{H}(V, F(V))+\rho_{H}(F(V), R(F V))<\sigma+\delta / 2<\delta .
\end{aligned}
$$

Since for $n=2$, the boundary $F(V), V \in L_{\delta}(n)$, does not contain an open subset of a sphere, $f_{2}(V)$ which is affinely equivalent $F(V)$, also does not contain any open subsets of the sphere. Therefore, $\operatorname{Im} f_{1} \cap \operatorname{Im} f_{2}=\emptyset$.

\section{Acknowledgements}

The preliminary version of this paper was written during the visit by the first author to the University of Ljubljana in June 1996 and announced in [6] and [7]. It was presented at the Second Iberoamerican Conference on Topology and Its Applications in Morelia, Mexico, in March 1997 [3] and at the General and Geometric Topology Conference in Kyoto, Japan, in March 1998 [23]. The first author acknowledges the support of INTAS grant No. 96-0712. The second author acknowledges the support of the Ministry for Science and Technology of the Republic of Slovenia grant No. J10885-0101-98. The authors thank the referee for several comments.

\section{References}

[1] H. Abels, 'Parallelizability of proper actions, global $K$-slices and maximal compact subgroups', Math. Ann. 212 (1974), 1-19.

[2] — 'Universal proper G-spaces', Math. Z. 159 (1978), 143-158.

[3] S. M. Ageev, 'Theory of extensors for non-compact Lie groups', in: Abstr. II Congr. Iberoamer. Topol. Appl., (Math. Inst. UNAM Morelia, Mexico, 1997) p. 11.

[4] S. M. Ageev and S. A. Bogati1̌, 'On nonhomeomorphicity of the Banach-Mazur compactum to the Hilbert cube', Uspehi Mat. Nauk 53 (1998), 209-210; English translation: Russian Math. Surveys 53 (1998), 205-207.

[5] S. M. Ageev, S. A. Bogatir and P. Fabel, 'The Banach-Mazur compactum $Q(n)$ is an AR', Vestnik MGU Mat. Meh. 1 (1998), 11-13; English translation: Moscow Univ. Math. Bull. 53 (1998), 10-12.

[6] S. M. Ageev, S. A. Bogatĭ̌, P. Fabel and D. Repovš, 'Extensors of noncompact Lie groups', Dokl. Ross. Akad. Nauk 362 (1998), 151-154; English translation: Dokl. Math. 58 (1998), 190-193.

[7] S. M. Ageev and D. Repovš, 'Theory of equivariant absolute extensors for noncompact Lie groups', preprint, University of Ljubljana, 1997.

[8] S. A. Antonyan, 'Retraction properties of an orbit space', Mat. Sbor. 137 (1988), 300-318; English translation: Math. USSR Sbor. 65 (1990), 305-321.

[9] J.-P. Aubin and H. Frankowska, Set-valued analysis (Birkhäuser, Basel, 1990).

[10] R. H. Bing, 'Partitioning continuous curves', Bull. Amer. Math. Soc. 58 (1952), 536-556.

[11] D. W. Curtis, 'Some theorems and examples on local equiconnectedness and its specializations', Fund. Math. 72 (1971), 101-113. 
[12] C. H. Dowker, 'Homotopy extension theorems', Proc. London Math. Soc. 6 (1956), 100-116.

[13] R. Engelking, General topology (Heldermann, Berlin, 1989).

[14] P. Fabel, 'The Banach-Mazur compactum $Q(2)$ is an AR', in: Abstracts Borsuk-Kuratowski session (Warsaw 1996) p. 5.

[15] W. E. Haver, 'Locally contractible spaces that are absolute neighbourhood retracts', Proc. Amer. Math. Soc. 40 (1973), 280-284.

[16] J. Jaworowski, 'Symmetry products of ANR's', Math. Ann. 192 (1971), 173-176.

[17] F. John, Extremum problems with inequalities as subsidiary conditions, Studies and Essays, Courant Anniversary Volume (Interscience, New York 1948) pp. 187-204.

[18] S. Mardešić, 'Approximate polyhedra, resolutions of maps and shape fibrations', Fund. Math. 114 (1981), 53-78.

[19] J. van Mill, Infinite-dimensional topology: prerequisites and introduction, North-Holland Math. Library 43 (North-Holland, Amsterdam, 1989).

[20] M. Murayama, 'On G-AN R's and their G-homotopy types', Osaka J. Math. 20 (1983), 479-512.

[21] R. S. Palais, 'The classification of G-spaces', Mem. Amer. Math. Soc. 36 (1960).

[22] — 'On the existence of slices for actions of non-compact Lie groups', Ann. of Math. (2) 73 (1961), 295-323.

[23] D. Repovš, 'Geometric topology of Banach-Mazur compacta', in: General and geometric topology (Japanese) (Kyoto, 1998) Surikaisekikenkyusho Kokyuroku 1074 (1999), 89-101.

[24] D. Repovš and P. V. Semenov, Continuous selections of multivalued mappings (Kluwer, Dordrecht, 1998).

[25] J. H. C. Whitehead, 'Note on a theorem due to Borsuk', Bull. Amer. Math. Soc. 54 (1948), $1125-1132$.

Department of Mathematics

Brest State University

Brest 224665

Belorussia

e-mail: ageev@kiipm.belpak.brest.by
Department of Mathematics

University of Ljubljana

Ljubljana 1001

Slovenia

e-mail: dusan.repovs@fmf.uni-lj.si 